\title{
Bioanalysis
}

\section{HRMS in DMPK}

\author{
"...HRMS provided precision, accuracy, specificity and sensitivity \\ comparable to what was obtained from the SRM assays for the same \\ drugs."
} First draft submitted: 17 June 2016; Accepted for publication: 27 June 2016;
Published online: 3 August 2016

Keywords: drug metabolism and PK • high-resolution MS $\bullet$ LC-MS $\bullet$ SRM $\bullet$ SWATH

The goal of the current drug metabolism and PK (DMPK) science is to enable the discovery and development of safe and efficacious new molecular entities within a reasonable time and at an acceptable cost. The impact of MS technology on DMPK science is unequivocal [1]. Interest in using high-resolution MS (HRMS) continues to expand in the bioanalysis arena as well as other pharmaceutical applications [2-9]. In addition to DMPK applications based on small molecules [10-12], HRMS is also being utilized for large molecules including proteins and peptide therapeutics [13-15].

Henry et al. [16] reported on a detailed comparison of HRMS assays to SRM assays using a triple quadrupole mass spectrometer for 17 therapeutic drugs measured in three different validated SRM assays. The comparison concluded that HRMS provided precision, accuracy, specificity and sensitivity comparable to what was obtained from the SRM assays for the same drugs. HRMS was found to have the advantage that SRM method development was not needed for the assays. In addition, HRMS was found to provide useful sample troubleshooting information that would not have been available from the SRM assay of the same sample [16].

Another major capability of HRMS is that it has the potential to provide both quantitative and qualitative (quan/qual) data. In the recent article by King et al. [9], the authors concluded that while current HRMS systems can provide bioanalytical data that are equivalent to data from triple quadrupole systems, there are processes that need to be implemented in order to successfully enable a quantitative/qualitative (quan/qual) strategy for a DMPK group. The processes suggested by King et al. [9] include the following: ensure that the new quan/qual workflow does not compromise the data quality or throughput of the previous systems; minimize the changes in the working practices needed to implement the quan/qual workflow; ensure that personnel needed to implement the quan/qual approach have experience in both areas; design a clear strategy to define which studies will be assayed using the quan/qual approach; ensure that the MS and data processing software packages are able to provide the expected results in the time allowed for the process and ensure that the local IT systems can handle the increased large data files that will be produced by the MS system. The authors also noted that MS vendors as well as third party software vendors need to improve the quan/qual data software packages.

This special issue of Bioanalysis focused on HRMS is a follow-up to the two special focus issues of Bioanalysis on HRMS that were published in 2012 and 2013. The 2012 publications focused primarily on discovery bioanalysis plus metabolite identification applications of HRMS. The 2013 publications expanded the focus to include regulated bioanalysis, peptide quantitation and protein therapeutics as well as editorials and opinions

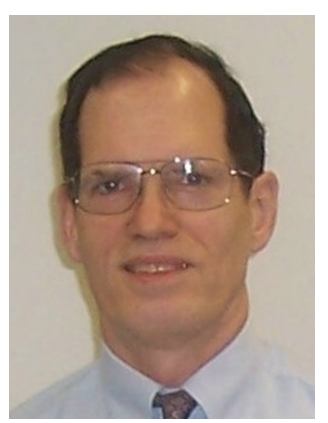

Walter Korfmacher Drug Metabolism \& Pharmacokinetics, Sanofi, Waltham, MA, USA and PDM-NCE, Pfizer World Wide Research \& Development, Groton, CT, USA

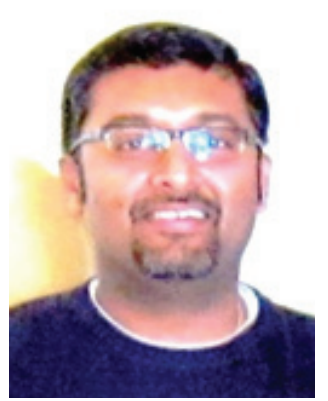

Ragu Ramanathan Author for correspondence: PDM-NCE, Pfizer World Wide Research \& Development, Groton, CT, USA ragu.ramanathan@pfizer.com 
on various HRMS topics including using HRMS for forensic and clinical toxicology.

The 2016 special issue of Bioanalysis, which focuses on HRMS, is a collection of articles that updates and expands the scope of how HRMS is being used by scientists engaged in various aspects of new drug discovery and drug development. This issue includes an opinion on HRMS as well as a commentary on HRMS $[17,18]$. The opinion discusses the rationales between the abbreviations/terminology HRMS and high-resolution accurate MS. The commentary provides readers some directions regarding outsource ability of HRMS-based DMPK studies and contract research organizations with HRMS capabilities. This is followed by a preliminary communication showing how HRMS was used in a metabolism study [19]. The communication details how data-independent sequential windowed acquisition of all theoretical fragments-based HRMS technology is used to solve a long-standing metabolite formation question. A perspective on how, when and why HRMS is implemented in regulated bioanalysis is discussed by Strum et al. [20]. Wei [21] and Weng [22] expand the

\section{References}

1 Korfmacher WA. Principles and applications of LC-MS in new drug discovery. Drug Discov. Today 10(20), 1357-1367 (2005)

2 Xian F, Hendrickson CL, Marshall AG. High resolution mass spectrometry. Anal. Chem. 84(2), 708-179 (2012).

3 Ramanathan R, Korfmacher W. The emergence of high-resolution MS as the premier analytical tool in the pharmaceutical bioanalysis arena. Bioanalysis 4(5), 467-469 (2012).

4 Ramanathan R, Jemal M, Ramagiri S et al. It is time for a paradigm shift in drug discovery bioanalysis: from SRM to HRMS. J. Mass Spectrom. 46(6), 595-601 (2011).

5 Korfmacher W. High-resolution mass spectrometry will dramatically change our drug-discovery bioanalysis procedures. Bioanalysis 3(11), 1169-1171 (2011).

6 Ramanathan DM. Looking beyond the SRM to highresolution MS paradigm shift for DMPK studies. Bioanalysis 5(10), 1141-1143 (2013).

7 Huang MQ, Lin ZJ, Weng W. Applications of highresolution MS in bioanalysis. Bioanalysis 5(10), 1269-1176 (2013).

8 Fung EN, Jemal M, Aubry AF. High-resolution MS in regulated bioanalysis: where are we now and where do we go from here? Bioanalysis 5(10), 1277-1284 (2013).

9 King L, Kotian A, Jairaj M. Introduction of a routine quan/qual approach into research DMPK: experiences and evolving strategies. Bioanalysis 6(24), 3337-3348 (2014).

10 Zelesky V, Schneider R, Janiszewski J, Zamora I, Ferguson J, Troutman M. Software automation tools for increased throughput metabolic soft-spot identification in early drug discovery. Bioanalysis 5(10), 1165-1179 (2013). applications of HRMS-based assays into antibodydrug conjugates and therapeutic proteins, respectively. This issue also has a research article on improved background subtraction when using HRMS [23]. Finally, there are two review articles in this special issue; one discusses high-throughput discovery in vitro ADME assays based on HRMS [24] and the other describes the sequential windowed acquisition of all theoretical fragments acquisition mode for DMPK and metabolomics studies [25]. In summary, this special issue contains multiple articles that will be of great interest to those using HRMS now or in the near future.

\section{Financial \& competing interests disclosure}

The authors have no relevant affiliations or financial involvement with any organization or entity with a financial interest in or financial conflict with the subject matter or materials discussed in the manuscript. This includes employment, consultancies, honoraria, stock ownership or options, expert testimony, grants or patents received or pending, or royalties.

No writing assistance was utilized in the production of this manuscript.

11 Ma S, Chowdhury SK. Data acquisition and data mining techniques for metabolite identification using LC coupled to high-resolution MS. Bioanalysis 5(10), 1285-1297 (2013).

12 Grondin A, Pillon A, Vandenberghe I, Guilbaud N, Kruczynski A, Gomes B. Discovery bioanalysis and in vivo pharmacology as an integrated process: a case study in oncology drug discovery. Bioanalysis 8(14), 1481-1498 (2016) .

13 Wei H, Tymiak AA, Chen G. High-resolution MS for structural characterization of protein therapeutics: advances and future directions. Bioanalysis 5(10), 1299-1313 (2013)

14 Morin LP, Mess JN, Garofolo F. Large-molecule quantification: sensitivity and selectivity head-to-head comparison of triple quadrupole with Q-TOF. Bioanalysis 5(10), 1181-1193 (2013).

15 Dillen L, Cools W, Vereyken L et al. Comparison of triple quadrupole and high-resolution TOF-MS for quantification of peptides. Bioanalysis 4(5), 565-579 (2012).

16 Henry H, Sobhi HR, Scheibner O, Bromirski M, Nimkar $\mathrm{SB}$, Rochat B. Comparison between a high-resolution single-stage Orbitrap and a triple quadrupole mass spectrometer for quantitative analyses of drugs. Rapid Commun. Mass Spectrom. 26(5), 499-509 (2012).

17 Ramanathan R, Korfmacher W. HRMS or HRAMS Bioanalysis 8(16), 1639-1640 (2016).

18 Rago B, Negahban A. Outsource-ability of HRMS-based DMPK Assays. Bioanalysis 8(16), 1641-1644 (2016).

19 Aratyn-Schaus Y, Ramanathan R. Advances in high resolution mass spectrometry and hepatocyte models solve a long-standing metabolism challenge: the loratadine story. Bioanalysis 8(16), 1645-1662 (2016). 
20 Sturm RM. High resolution accurate mass spectrometry for quantitative regulated bioanalysis: how, when, and why to implement. Bioanalysis 8(16), 1709-1721 (2016).

21 Grafmuller L, Wei C, Ramanathan R, Barletta F, Steenwyk $\mathrm{R}$, Tweed J. Unconjugated payload quantification and DAR characterization of antibody-drug conjugates using high resolution mass spectrometry (HRMS). Bioanalysis $8(16)$, 1663-1678 (2016).

22 Weng N. A workflow for absolute quantitation of large therapeutic proteins in biological samples at intact level using LC-HRMS. Bioanalysis 8(16), 1679-1691 (2016).
23 Chowdhury S. Development of an accelerated background subtraction algorithm for processing high resolution mass spectrometry data. Bioanalysis 8(16), 1693-1707 (2016).

24 Shou WZ. Recent developments in software and automation tools for high-throughput discovery in vitro ADME assays based on HRMS. Bioanalysis 8(16), 1723-1733 (2016).

25 Hopfgartner G. SWATH acquisition mode for drug metabolism and metabolomics investigations. Bioanalysis 8(16), 1735-1750 (2016). 